\title{
Cochlear Implantation in Inner Ear Malformations: Considerations Related to Surgical Complications and Communication Skills
}

\author{
Recep Karamert ${ }^{a} \quad$ Hakan Tutar $^{a}$ Şenay Altinyay ${ }^{b}$ Mehmet Düzlü ${ }^{a}$ \\ Merve Yildiz $^{\mathrm{a}}$ İsmail Akdulum ${ }^{c}$ Mehmet Birol Uğur ${ }^{\mathrm{a}}$ Mustafa Çolak $^{\mathrm{a}}$ \\ Süleyman Cebeci ${ }^{a}$ Muammer Melih Şahin ${ }^{a}$ Elçin Orçan ${ }^{b}$ \\ Yıldırım Ahmet Bayazit ${ }^{d}$

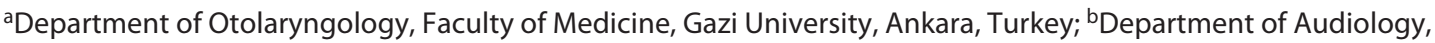 \\ Faculty of Medicine, Gazi University, Ankara, Turkey; ' Department of Radiology, Faculty of Medicine, Gazi University,

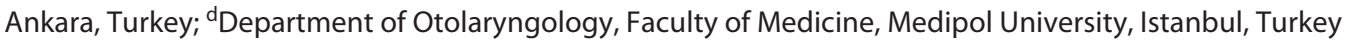

\section{Keywords \\ Cochlear implant · Inner ear malformation · Congenital hearing loss · Gusher}

\begin{abstract}
Introduction: There are particular challenges in the implantation of malformed cochleae, such as in cases of facial nerve anomalies, cerebrospinal fluid (CSF) leaks, erroneous electrode insertion, or facial stimulation, and the outcomes may differ depending on the severity of the malformation. The aim of this study was to assess the impact of inner ear malformations (IEMs) on surgical complications and outcomes of cochlear implantation. Methods: In order to assess the impact of IEMs on cochlear implant $(\mathrm{Cl})$ outcomes, 2 groups of patients with similar epidemiological parameters were selected from among 863 patients. Both the study group (patients with an IEM) and control group (patients with a normal inner ear) included 25 patients who received a $\mathrm{Cl}$ and completed at least 1 year of follow-up. Auditory performance, receptive and expressive language skills, and production and use of speech were evaluated preoperatively and at
\end{abstract}

least 1 year after implantation. Types of surgical complications and rates of revision surgeries were determined in each group. Results: In the study group, the most common malformation was an isolated enlarged vestibular aqueduct (EVA) (44.8\%). Overall, the patients with IEMs showed significant improvement in auditory-verbal skills. In general, the patients who had normal cochleae scored significantly better compared to patients with IEMs $(p<0.05)$. The complication rate was significantly lower in the control group compared to the study group ( $p=0.001)$, but the rate of revision surgeries did not differ significantly ( $p=0.637)$. Conclusion: It is possible to improve communication skills with $\mathrm{Cls}$ in patients with IEMs despite the variations in postoperative performances. Patients with EVA, incomplete partition type 2 , and cochlear hypoplasia type 2 were the best performers in terms of auditory-verbal skills. Patients with IEMs scored poorly compared to patients with normal cochleae. CSF leak (gusher or oozing) was the most common complication during surgery, which is highly likely in cases of incomplete partition type 3.

(c) 2021 S. Karger AG, Basel karger@karger.com www.karger.com/orl
(C) 2021 S. Karger AG, Basel

Karger ${ }^{\prime}=$
Correspondence to:

Recep Karamert, recepkaramert@gmail.com 


\section{Introduction}

Inner ear malformations (IEMs) are among the common causes of congenital hearing loss [1]. After the description of the specific malformation consisting of a cochlear duct shortened to $11 / 2$ turns with a defective apical modiolus and interscalar septa by Mondini [2], the term "Mondini's dysplasia" has been widely used to describe any kind of malformation of the bony labyrinth.

With the advances in radiological imaging over the last decades, new imaging techniques have revealed a wide variety of anomalies of the bony labyrinth. Jackler et al. [1] described the first classification of congenital malformations of the inner ear, which were detected by radiologic imaging. They claimed that the type and severity of IEMs depend on the arrested stage of embryogenesis in a linear developmental model. A further detailed classification based on this theory was made by Sennaroglu and Saatci [3]. Papsin [4] negated this single linear developmental model and suggested that there might be multiple and independent pathways leading to different malformations.

There are particular challenges in the implantation of malformed cochleae when placing a cochlear implant (CI). The facial nerve may have an aberrant course, and care must be taken to avoid injuries of the fallopian canal and the nerve. Aberrant course of the labyrinthine segment in particular malformations may result in facial stimulation with the CI. Cerebrospinal fluid (CSF) leaks are also common in cases of IEMs. Intraoperative management of the leakage may be bothersome, and inadequate interventions may lead to severe postoperative intracranial infectious complications. Implantation of a cochleawith severeinternalbonyarchitecturemalformations may result in an erroneous electrode insertion into the internal acoustic canal or vestibular portion. Furthermore, outcomes may vary depending on the severity of the malformation $[1,5]$. Although IEMs were considered as contraindications for cochlear implantation in the early cochlear implant era, today it is widely accepted that satisfactory results can be obtained with CIs in patients with IEMs $[4,6,7]$. About a quarter of such cases are complicated because of the malformed anatomy or CSF leaks (gusher or oozing), but major complications are rare, and the rate of revision surgeries is comparable to that seen among patients who have normal inner ear anatomy [4, 8]. This is a case-control study in which patients with IEMs were matched to normal controls in order to assess the impact of IEMs on the surgical complications and outcomes of CIs.

\section{Materials and Methods}

Medical records of the patients who received a CI or auditory brainstem implant between July 2002 and September 2019 were reviewed retrospectively after ethics committee approval was obtained from the university. The radiologic images (CT and MRI) of 863 patients were recruited and evaluated regarding IEMs as described previously [5].

In order to assess the impact of IEMs on CI outcomes, 2 groups of patients were selected from among those 863 patients. Inclusion criteria for the study group were having an IEM, receiving unilateral or bilateral CIs in malformed ears, and completing at least 1 year of follow-up. Overall, an IEM was detected in 84 ears of 43 (5\%) patients from among 863 patients. Of these, 25 patients fulfilled all inclusion criteria. A total of 29 CIs were applied for these 25 patients. The second group comprised 25 patients with normal inner ears who received a CI and completed at least 1 year of follow-up. A total of 29 CIs were applied for these 25 controls. The control patients were selected because their epidemiological parameters were comparable to those of the patients with IEMs.

The audiological performance of the 2 groups was compared. The Meaningful Auditory Integration Scale (MAIS), LittleEARS, and Category of Auditory Performance (CAP) tests were administered to assess auditory performance. A validated native language version of the Test of Early Language Development-3rd edition (TELD-3) and the Turkish Expressive and Receptive Language Test (TIFALDI) were administered to assess the receptive and expressive language skills of the patients preoperatively and at least 1 year after the implantation. The Meaningful Use of Speech Scale (MUSS) and Speech Intelligibility Rating (SIR) tests were administered to assess production and use of speech.

Factors that could influence the post-implantation performance of the patients such as age at implantation, duration of implant use, family involvement, and presence of accompanying handicaps were also assessed. Family involvement was scored according to the rating system described by Moeller [9]. Accompanying handicaps were determined as developmental disorders, mental retardation, and genetic syndromes that may impair auditory-verbal performance.

\section{Statistical Analysis}

SPSS 22.0 (IBM Corp., Armonk, NY, USA) was used for statistical analyses. The variables were tested for normal distribution using the Kolmogorov-Smirnov test and histograms. Data were presented as mean \pm standard deviation and percentage. The $\chi^{2}$ test was used to compare categorical data. The independent samples $t$ test or 1-way ANOVA and the Mann-Whitney U test or KruskalWallis test were used to compare parametric and nonparametric data, respectively. Preoperative and postoperative measures were compared using the Wilcoxon signed rank test. Correlation analysis was performed using Pearson and Spearman correlation tests. In all tests, $p<0.05$ was considered as statistical significance.

\section{Results}

Overall, an IEM was detected in 84 ears of 43 (5\%) patients among 863 patients. The most common malformation detected was an enlarged vestibular aqueduct (EVA), 
seen in 38 of 84 ears (45\%). An isolated EVA was seen in $18(21.4 \%)$ ears. A second malformation accompanied the EVA in 10 (11.9\%) ears. An incomplete partition (IP) was seen in 35 of 84 ears (41.6\%). Common cavity (CC) and cochlear hypoplasia $(\mathrm{CH})$ were seen in $5.9 \%$ and $7.1 \%$ of the patients with IEMs, respectively.

In both the study and control groups, there were 12 (48\%) males and 13 (52\%) females. The ages ranged from 2 to 16 (mean: 9.4) and 3 to 19 (mean: 9.02) years in the study and control groups, respectively. Age at implantation ranged from 1 to 12 years in both groups, with a mean implantation age of 4.8 years and 4.72 years in the study and control groups, respectively. The implantation was unilateral in 21 (84\%) patients in both groups. Four (16\%) patients received bilateral implants in both groups. Duration of implant use was between 1 and 10 years in both groups, with a mean of 4.6 and 4.3 years in the study and control groups, respectively.

There was no significant difference between control and study groups regarding demographic features, gender, side and age of implantation, or duration of implant use $(p>0.05)$. There was no significant relationship between gender and type of malformation $(p=0.227)$. There was no significant correlation between age of implantation and postoperative outcomes of the auditory, speech, or language tests $(p>0.05)$.

In the study group, the most common malformation was isolated EVA (44.8\%) (Table 1). Overall, the patients with IEMs showed significant improvement in auditory performance, receptive and expressive language skills, and production and use of speech (Table 2). The patients with incomplete partition type 2 (IP-2), cochlear hypoplasia type $2(\mathrm{CH}-2)$, and EVA achieved the best scores while incomplete partition type 3 (IP-3) patients showed moderate progress. The worst outcomes were seen in cases of cochlear hypoplasia type $4(\mathrm{CH}-4)$, CC, incomplete partition type 1 (IP-1), and narrow internal auditory canal (IAC) (Table 3).

In general, the patients who had normal cochleae scored significantly better compared to patients with IEMs $(p<0.05)$. However, although the postoperative scores of patients with EVA, CH-2, and IP-2 were usually comparable to the scores of the patients with normal cochleae, the normal cochlea group was superior to EVA patients in terms of expressive language skills $(p=0.03)$ and MUSS $(p=0.019)$ and CAP $(p<0.001)$ scores (Table 4).

In the study group, 12 (48\%) patients had an accompanying handicap that might impair postoperative performance. Eight (32\%) patients had one of the pervasive

Cochlear Implantation in Inner Ear

Malformations
Table 1. Types of inner ear malformations detected in 29 implanted ears of 25 patients

\begin{tabular}{lll}
\hline Malformation & $n$ & $\%$ \\
\hline IP-1 & 4 & 13.7 \\
IP-2 & 2 & 6.8 \\
IP-3 & 4 & 13.7 \\
EVA & 13 & 44.8 \\
CC & 1 & 3.4 \\
CH-2 & 3 & 10.3 \\
CH-4 & 1 & 3.4 \\
Narrow IAC & 1 & 3.4 \\
\hline Total & 29 & 100.0
\end{tabular}

IP, incomplete partition; $\mathrm{CH}$, cochlear hypoplasia; CC, common cavity; EVA, enlarged vestibular aqueduct; IAC, internal auditory canal.

developmental disorders, autism spectrum disorders, mental retardations, and motor disabilities or developmental disabilities. Four (16\%) patients had fragile-X syndrome, Phelan-McDermid syndrome, CHARGE syndrome, and mitochondrial myopathy, respectively. There was no significant relationship between the type of malformation and presence of an accompanying handicap $(p=0.14)$. The presence of an accompanying handicap did not correlate with the postoperative audiological scores in the study group except for the MUSS scores, which were significantly worse among the handicapped patients $(p=0.022)$.

In the control group, 7 (28\%) patients had mild or moderate mental retardation. This accompanying handicap negatively affected postoperative CAP and SIR scores and receptive and expressive language skills $(p=0.003$, $p=0.001, p=0.004$, and $p=0.015$, respectively). Although accompanying handicaps were less common in the control group than the study group, this difference did not reach statistical significance $(p=0.145)$.

There was no significant difference between the family involvement scores of the study and control groups $(p=0.307)$. Family involvement scores for different malformations were also not significantly different $(p=$ $0.219)$. There was no relationship between family involvement scores and postoperative auditory performance scores in the study group ( $p>0.05)$. However, in the control group, there was a significant correlation between family involvement scores and postoperative CAP and SIR scores ( $p=0.004$ and $p=0.003$, respectively). The complication rate was significantly lower in the control 
Table 2. Pre- and post-cochlear implant auditory performance and communication skills in patients with malformed inner ears

\begin{tabular}{|c|c|c|c|c|}
\hline Evaluation & Preop (mean) & Postop (mean) & Std. deviation & $p$ value \\
\hline \multicolumn{5}{|l|}{ Auditory performance } \\
\hline MAIS & 8.78 & 28.84 & 12.65 & $<0.001 *$ \\
\hline LittleEARS & 2.58 & 20.68 & 13.92 & $<0.001 *$ \\
\hline CAP & 0.59 & 3.64 & 2.02 & $<0.001 *$ \\
\hline \multicolumn{5}{|c|}{ Production and use of speech } \\
\hline MUSS & 6 & 17.89 & 9.93 & $<0.001^{*}$ \\
\hline SIR & 1.29 & 2.83 & 1.42 & $<0.001 *$ \\
\hline \multicolumn{5}{|l|}{ Language skills } \\
\hline Receptive language & 15.71 & 34.13 & 20.49 & $0.001 *$ \\
\hline Expressive language & 14.38 & 30.52 & 18.9 & $0.001 *$ \\
\hline
\end{tabular}

MAIS, Meaningful Auditory Integration Scale; CAP, Category of Auditory Performance; MUSS, Meaningful Use of Speech Scale; SIR, Speech Intelligibility Rating.

Table 3. Comparison of the postoperative scores between IEMs

\begin{tabular}{llllllllll}
\hline & $\begin{array}{l}\mathrm{IP}-1 \\
(n=4)\end{array}$ & $\begin{array}{l}\mathrm{IP}-2 \\
(n=2)\end{array}$ & $\begin{array}{l}\mathrm{IP}-3 \\
(n=4)\end{array}$ & $\begin{array}{l}\mathrm{EVA} \\
(n=10)\end{array}$ & $\begin{array}{l}\mathrm{CC} \\
(n=1)\end{array}$ & $\begin{array}{l}\mathrm{CH}-2 \\
(n=2)\end{array}$ & $\begin{array}{l}\mathrm{CH}-4 \\
(n=1)\end{array}$ & $\begin{array}{l}\text { N. IAC } \\
(n=1)\end{array}$ & $p$ value \\
\hline $\begin{array}{l}\text { Auditory performance } \\
\quad \text { MAIS }\end{array}$ & 8 & 40 & 27 & 34.3 & 21 & 39 & 18 & 9 & $\mathbf{0 . 0 2 1 *}$ \\
$\quad \begin{array}{l}\text { L.EARS } \\
\quad \text { CAP }\end{array}$ & 3 & 35 & 13 & 28.2 & 15 & 23 & 10 & 3 & 0.151 \\
$\begin{array}{l}\text { Production and use of speech } \\
\quad \text { MUSS }\end{array}$ & 2 & 6 & 2.5 & 3.7 & 4 & 5 & 3 & 1 & 0.307 \\
$\quad$ SIR & 6.5 & 31 & 15.2 & 21.2 & 10 & 22.5 & 11 & 10 & 0.308 \\
$\begin{array}{l}\text { Language skills } \\
\quad \text { Receptive }\end{array}$ & 1.5 & 4.5 & 2 & 3.3 & 2 & 2.5 & 2 & 1 & 0.187 \\
$\quad$ Expressive & 32.3 & 45 & 36 & 38 & 21 & 34.5 & 21 & 10 & 0.932 \\
\hline
\end{tabular}

IP, incomplete partition; $\mathrm{CH}$, cochlear hypoplasia; CC, common cavity; EVA, enlarged vestibular aqueduct; IAC, internal auditory canal; IEM, inner ear malformation; MAIS, Meaningful Auditory Integration Scale; CAP, Category of Auditory Performance; MUSS, Meaningful Use of Speech Scale; SIR, Speech Intelligibility Rating.

group compared to the study group ( $p=0.001$ ), but the rate of revision surgeries did not differ significantly $(10.3 \%$ and $6.8 \%$ in the study and control groups, respectively; $p=0.637$ ).

In the study group, the complication and revision rates were $48.2 \%$ and $10.3 \%$, respectively. The most common complication in the study group was CSF leak, which was observed in $13(44.8 \%)$ of 29 implanted ears. This was in the form of a gusher or oozing in $9(31 \%)$ and $4(13.7 \%)$ ears, respectively. All CSF leaks were controlled intraoperatively by soft tissue sealing without lumbar drainage. Three patients needed revision surgery due to recurrence of the CSF leak, two with EVA and one with IP-1. A peri- lymph gusher was observed in all IP-3 cases and 2 of 4 (50\%) IP-1 cases, and EVA patients presented either with a gusher or oozing equally in 6 of 13 ears $(46.1 \%)$. In 1 patient (3.4\%) with $\mathrm{CH}-4$, transient facial palsy occurred, which recovered spontaneously during follow-up.

In the control group, 2 (6.8\%) complications were encountered. One patient with an early electrode array migration was re-treated, and the electrode array was reinserted into the cochlea. The other patient with flap breakdown in the second month was re-treated, the CI was explanted, and a new device was implanted to the contralateral side. 
Table 4. Pre- and post-cochlear implant auditory performance and communication skills in patients with malformed cochleae versus normal inner ears

\begin{tabular}{|c|c|c|c|c|c|c|}
\hline \multirow[t]{2}{*}{ Parameter } & \multirow[t]{2}{*}{ Evaluation test } & \multirow{2}{*}{$\begin{array}{l}\text { Inner ear } \\
\text { morphology }\end{array}$} & \multicolumn{2}{|l|}{ Pre-implant } & \multicolumn{2}{|l|}{ Post-implant } \\
\hline & & & mean score & $p$ value & mean score & $p$ value \\
\hline \multirow[t]{3}{*}{$\begin{array}{l}\text { Auditory } \\
\text { performance }\end{array}$} & MAIS & $\begin{array}{l}\text { Malformed } \\
\text { Normal }\end{array}$ & $\begin{array}{l}8.7 \\
13.6 \\
\end{array}$ & 0.18 & $\begin{array}{l}28.8 \\
36.4\end{array}$ & $0.001 *$ \\
\hline & LittleEARS & $\begin{array}{l}\text { Malformed } \\
\text { Normal }\end{array}$ & $\begin{array}{l}2.5 \\
9.6\end{array}$ & $0.04 *$ & $\begin{array}{l}20.6 \\
31.6\end{array}$ & $0.003^{*}$ \\
\hline & CAP & $\begin{array}{l}\text { Malformed } \\
\text { Normal }\end{array}$ & $\begin{array}{l}0.5 \\
2.2\end{array}$ & $0.01 *$ & $\begin{array}{l}3.6 \\
5.9\end{array}$ & $0.001 *$ \\
\hline \multirow[t]{2}{*}{$\begin{array}{l}\text { Production and } \\
\text { use of speech }\end{array}$} & MUSS & $\begin{array}{l}\text { Malformed } \\
\text { Normal }\end{array}$ & $\begin{array}{l}6 \\
10.2 \\
\end{array}$ & 0.9 & $\begin{array}{l}17.8 \\
32.2\end{array}$ & $0.001 *$ \\
\hline & SIR & $\begin{array}{l}\text { Malformed } \\
\text { Normal }\end{array}$ & $\begin{array}{l}1.2 \\
2\end{array}$ & $0.01 *$ & $\begin{array}{l}2.8 \\
4.1\end{array}$ & $0.001 *$ \\
\hline \multirow[t]{2}{*}{ Language skills } & $\begin{array}{l}\text { Receptive } \\
\text { language }\end{array}$ & $\begin{array}{l}\text { Malformed } \\
\text { Normal }\end{array}$ & $\begin{array}{l}15.7 \\
21.4\end{array}$ & 0.25 & $\begin{array}{l}34.1 \\
51.2\end{array}$ & $0.003^{*}$ \\
\hline & $\begin{array}{l}\text { Expressive } \\
\text { language }\end{array}$ & $\begin{array}{l}\text { Malformed } \\
\text { Normal }\end{array}$ & $\begin{array}{l}14.3 \\
20.9\end{array}$ & 0.14 & $\begin{array}{l}30.5 \\
48.8\end{array}$ & $0.001 *$ \\
\hline
\end{tabular}

MAIS, Meaningful Auditory Integration Scale; CAP, Category of Auditory Performance; MUSS, Meaningful Use of Speech Scale; SIR, Speech Intelligibility Rating.

\section{Discussion}

Detection of an IEM is not uncommon in cases of congenital hearing loss $[1,4,10]$. Patients with IEMs are expected to perform worse with a CI compared to patients with normal cochleae $[10,11]$. However, it was also suggested that outcomes of CIs in cases of normal cochleae and IEMs would be comparable except for CC $[12,13]$. Despite these controversies, it is generally accepted that outcomes depend on the severity of the IEM, and that postoperative communication skills are worse as the severity of IEM increases $[1,4,6,11]$. Patients with relatively mild malformations like EVA and IPs are usually the best performers, whereas those with $\mathrm{CC}, \mathrm{CH}$, and narrow IAC exhibit limited improvements $[4,6,7,11]$. According to our results, the patients with IEMs could benefit from their CIs and showed significant improvements in their communication skills, although their outcomes were significantly worse when compared to the patients with normal cochleae, except for cases of IP-2, CH-2, and EVA.

Since first described by Valvassori and Clemis [14], EVA has been the most common IEM detected $[4,10,15]$. Papsin [4] defined EVA as the most subtle detectable IEM and suggested the categorization of EVA as the mildest form of IP as evidenced by the study of Lemmerling et al. [16], who demonstrated a cochlear modiolus deficiency in all cases of EVA on CT scans. Patients with isolated EVA usually present with progressive hearing loss and generally have sufficient time for auditory-verbal development. Therefore, EVA patients are predicted to perform well with CIs due to this progressive nature of their hearing loss, which also allows them to gain some auditory-verbal experience $[11,17,18]$. These data support our results.

IP deformities are characterized by a relatively normal outline and internal architecture anomalies of the cochlea. The vestibular system and the cochlea are clearly separated from each other [5]. IP-1, also known as cystic cochleovestibular malformation, is the less differentiated malformation of this group. The internal bony architecture is severely defective, and neither the modiolus nor interscalar septa is observed in the cochlea. IP-2 is characterized by the lack of modiolus at the apical part of the cochlea with intact interscalar septa, which corresponds to the classical Mondini deformity and has the most preserved internal architecture among IPs. The IEM also encountered in X-linked deafness was defined as IP-3 [15]. Radiological features of this mal- 
formation are complete absence of the entire modiolus, a wide bulbous IAC, and incomplete separation of the coils of the cochlea from the IAC with preserved interscalar septa $[15,19]$. These patients can present with mixed hearing loss at the beginning, but the majority of them become candidates for cochlear implantation due to the progressive nature of their hearing loss. This is the rarest form of IPs at a rate of $2 \%$ [5]. The rates of different types of IPs were similar in our series.

Patients with IPs are claimed to have good postoperative performances with CIs [4]. However, most of the studies in the literature do not make a distinction between subgroups of IPs $[4,10,11]$. Although good outcomes are reported for IP-2 patients by several authors [6, 20, 21], there are a limited number of publications reporting the results for IP-1 and IP-3 [22, 23]. Outcomes varied markedly among IPs in our series. The IP- 2 patients were the best performers and even scored as good as the patients with normal cochleae. IP-3 patients showed moderate development despite the progressive nature of their hearing loss and preoperative auditory-verbal experience. IP-1 patients scored the worst among the IP cases in our series.

$\mathrm{CC}$ is a severe deformity in which the cochlea and vestibule form a single cavity without an internal architecture. Semicircular canals may be normal or malformed [1]. It is claimed that neural elements of both the auditory and vestibular systems lie on the outer wall of the cavity. CC constitutes $7.7-10.4 \%$ of IEMs $[4,10,15]$. The rate of $5.9 \%$ in our series seems comparable with that reported in the literature. CC is considered to result in poorer performance when compared to the more differentiated anomalies $[4,6,11]$, but excellent outcomes are also reported in some cases of CC $[10,24]$. In our series, CC was associated with limited auditory-verbal improvement.

Jackler et al. [1] defined $\mathrm{CH}$ as a rudimentary cochlea ranging from a small diverticulum to a bud of several millimeters. $\mathrm{CH}$ is characterized by smaller external dimensions and is divided into subgroups according to the deformities of the internal architecture [5]. In $\mathrm{CH}-2$, also known as cystic hypoplastic cochlea, the modiolus and interscalar septa are defective, and there may be a wide connection with the IAC as the entire modiolus is absent, which may lead to a gusher or the misinsertion of the electrode. $\mathrm{CH}-4$ is the mildest malformation in this group. In $\mathrm{CH}-4$, the basal turn of the cochlea is preserved, but the middle and apical turns are hypoplastic [5]. Facial nerve anomalies and usually the anterior displacement of the second genu, which may complicate surgery, are common in $\mathrm{CHs}[4,5]$. The small size of the cochlea in cases of $\mathrm{CH}$ may be an obstacle to full insertion of the electrode array. In the present series, $\mathrm{CH}-2$ and $\mathrm{CH}-4$ were detected at rates of $4.7 \%$ and $2.3 \%$, respectively. In 1 case of $\mathrm{CH}-$ 4 , the dehiscent facial nerve was passing over the round window, and the patient had facial palsy postoperatively. An immediate exploration revealed epineural damage without a transection. The facial palsy recovered completely during follow-up. In general, CI performances in cases of $\mathrm{CH}$ are not satisfactory $[4,6]$. Despite this fact, our patients with $\mathrm{CH}-2$ achieved good postoperative scores. However, the patient with a milder malformation, $\mathrm{CH}-4$, had the worst outcome. This may be due to accompanying moderate mental retardation and motor disability in the patient with $\mathrm{CH}-4$ while the $\mathrm{CH}-2$ patients had no accompanying handicaps.

The IAC is considered narrow if its midpoint diameter is $<1.5 \mathrm{~mm}$ [25]. Several authors have considered a narrowed IAC as a contraindication to CIs due to the high incidence of an absent cochlear nerve [25, 26]. MRI evaluation may be helpful to clarify the presence of the auditory nerve in narrow IACs [27]. However, even if a cochlear nerve is not visible on MRI, it is suggested that some auditory fibers may project through the vestibular branch of the eighth cranial nerve [28]. A precise audiologic evaluation is fundamental in such cases to show any auditory sensations that indicate an intact auditory nerve. Patients with no sensation of sound even at high intensities are at high risk of having cochlear nerve aplasia. We had 1 patient with a narrow IAC who received a CI. MRI revealed only 2 nerve fibers in both IACs. Despite the improvements achieved to some extent after the CI, this patient was the worst performer among all implantees of these IEM cases regarding communication skills.

Accompanying handicaps that may impair higher brain functions have a negative influence on CI performance [29-31]. The patients with additional handicaps performed worse in all groups, with patients with IEM and those with normal cochleae being similarly affected.

Family involvement has a positive influence on longterm outcomes of CIs $[32,33]$. According to our results, family involvement is an important factor for the patient's development. This was apparent in the patients with normal cochleae. However, there was no significant relationship between parental participation scores and postoperative outcomes in the IEM group. This finding may be related to the limited number of patients included in the study or may suggest that IEMs have a significant impact on implantees, limiting the influence of familial support.

CSF leak is the most common complication encountered with CIs in cases of IEMs $[4,6,10,34]$. A CSF leak is likely to occur if there is dehiscence between the lateral 
fundus of the IAC and the cochlea $[4,35]$. CSF leaks are referred to as gushers or oozing according to the intensity of the flow. In our series, CSF leaks were observed in $44.8 \%$ of cases. A profuse gusher was observed in all IP-3 cases and $50 \%$ of IP- 1 cases. In EVA cases, the rate of CSF leak was almost $50 \%$, and both gushers and oozing were observed equally. CSF leaks were not observed in any of the patients with normal cochleae.

\section{Conclusion}

It is possible to improve communication skills with CIs in patients with IEMs despite the variations in postoperative performances. Patients with EVA, IP-2, and $\mathrm{CH}-2$ were the best performers while those with CC, IP-1, and narrow IAC were the worst performers in terms of auditory-verbal skills. The patients with IEMs scored poorly compared to the patients with normal cochleae. CSF leaks were the most common complication during surgery, being highly likely in cases of IP-3.

\section{Statement of Ethics}

All procedures performed in this study were in accordance with the 1964 Declaration of Helsinki and its later amendments or comparable ethical standards. Ethics committee approval was obtained from Gazi University (Reference No. 241/26.03.2018). Informed consent was not obtained because of the retrospective nature of the study.

\section{Conflict of Interest Statement}

The authors have no conflicts of interest to declare.

\section{Funding Sources}

There was no funding source for this study.

\section{Author Contributions}

Recep Karamert was involved in conception and design, data analysis, manuscript draft, revision of the manuscript, and approval of the final version and is accountable for all aspects of the work. Hakan Tutar was involved in conception and design, data analysis supervision, revision of the manuscript, and approval of the final version and is accountable for all aspects of the work. Şenay Altinyay was involved in data collection, revision of the manuscript, and approval of the final version and is accountable for all aspects of the work. Mehmet Düzlü was involved in data analysis, statistical analysis, revision of the manuscript, and approval of the final version and is accountable for all aspects of the work. Merve Yildiz was involved in data collection, revision of the manuscript, and approval of the final version and is accountable for all aspects of the work. İsmail Akdulum was involved in data analysis, revision of the manuscript, and approval of the final version and is accountable for all aspects of the work. Mehmet Birol Uğur was involved in conception and design, revision of the manuscript, and approval of the final version and is accountable for all aspects of the work. Mustafa Çolak was involved in data collection, revision of the manuscript, and approval of the final version and is accountable for all aspects of the work. Süleyman Cebeci was involved in data collection, revision of the manuscript, and approval of the final version and is accountable for all aspects of the work. Muammer Melih Şahin was involved in data collection, revision of the manuscript, and approval of the final version and is accountable for all aspects of the work. Elçin Orçan was involved in data collection, revision of the manuscript, and approval of the final version and is accountable for all aspects of the work. Yildirim Ahmet Bayazit was involved in conception and design, critical senior supervision of the study, revision of the manuscript, and approval of the final version and is accountable for all aspects of the work.

\section{References}

1 Jackler RK, Luxford WM, House WF. Congenital malformations of the inner ear: a classification based on embryogenesis. Laryngoscope. 1987;97:2-14

2 Mondini C. Anatomia surdi nedi section. De Bononiensi Sci Artum Inst Atque Acad Commentarii Bol. 1791;7(28):419.

3 Sennaroglu L, Saatci I. A new classification for cochleovestibular malformations. Laryngoscope. 2002;112:2230-41.

4 Papsin BC. Cochlear implantation in children with anomalous cochleovestibular anatomy. Laryngoscope. 2005;115:1-26.

5 Sennaroğlu L, Bajin MD. Classification and current management of inner ear malformations. Balkan Med J. 2017;34:397-411.
6 Farhood Z, Nguyen SA, Miller SC, Holcomb MA, Meyer TA, Rizk HG. Cochlear implantation in inner ear malformations: systematic review of speech perception outcomes and intraoperative findings. Otolaryngol Head Neck Surg. 2017;156:783-93.

7 Van Wermeskerken GK, Dunnebier EA, Van Olphen AF, Van Zanten BA, Albers FW. Audiological performance after cochlear implantation: a 2-year follow-up in children with inner ear malformations. Acta Otolaryngol. 2007;127:252-7.

8 Karamert R, Düzlü M, Tutar H, Eravcı FC, Türkcan AK, Zorlu ME, et al. Assessment of cochlear implant revision surgeries in a cohort of 802 patients. Otol Neurotol. 2019;40: $464-70$.
9 Moeller MP. Early intervention and language development in children who are deaf and hard of hearing. Pediatrics. 2000;106:E43.

10 Dettman S, Sadeghi-Barzalighi A, Ambett R, Dowell R, Trotter M, Briggs R. Cochlear implants in forty-eight children with cochlear and/or vestibular abnormality. Audiol Neurootol. 2011;16:222-32.

11 Buchman CA, Copeland BJ, Yu KK, Brown CJ, Carrasco VN, Pillsbury HC 3rd. Cochlear implantation in children with congenital inner ear malformations. Laryngoscope. 2004; 114:309-16. 
12 Arnoldner C, Baumgartner WD, Gstoettner W, Egelierler B, Czerny C, Steiner E, et al. Audiological performance after cochlear implantation in children with inner ear malformations. Int J Pediatr Otorhinolaryngol. 2004;68:457-67.

13 Eisenman DJ, Ashbaugh C, Zwolan TA, Arts HA, Telian SA. Implantation of the malformed cochlea. Otol Neurotol. 2001;22:83441.

14 Valvassori GE, Clemis JD. The large vestibular aqueduct syndrome. Laryngoscope. 1978; 88:723-8.

15 Sennaroglu L, Sarac S, Ergin T. Surgical results of cochlear implantation in malformed cochlea. Otol Neurotol. 2006;27:615-23.

16 Lemmerling MM, Mancuso AA, Antonelli PJ, Kubilis PS. Normal modiolus: CT appearance in patients with a large vestibular aqueduct. Radiology. 1997;204:213-9.

17 Au G, Gibson W. Cochlear implantation in children with large vestibular aqueduct syndrome. Am J Otol. 1999;20:183-6.

18 Aschendorff A, Marangos N, Laszig R. Large vestibular aqueduct syndrome and its implication for cochlear implant surgery. Am J Otol. 1997; 18:S57.

19 Phelps PD, Reardon W, Pembrey M, Bellman $S$, Luxom L. X-linked deafness, stapes gushers and a distinctive defect of the inner ear. Neuroradiology. 1991;33:326-30.

20 Munro KJ, George CR, Haacke NP. Audiological findings after multichannel cochlear implantation in patients with Mondini dysplasia. Br J Audiol. 1996;30:369-79.
21 Miyamoto RT, Robbins AJ, Myres WA, Pope ML. Cochlear implantation in the Mondini inner ear malformation. Am J Otol. 1986;7: $258-61$.

22 Incesulu A, Adapinar B, Kecik C. Cochlear implantation in cases with incomplete partition type III (X-linked anomaly). Eur Arch Otorhinolaryngol. 2008;265:1425-30.

23 Berrettini S, Forli F, De Vito A, Bruschini L, Quaranta N. Cochlear implant in incomplete partition type I. Acta Otorhinolaryngol Ital. 2013;33:56-62.

24 Ito J, Sakota T, Kato H, Hazama M, Enomoto M. Surgical considerations regarding cochlear implantation in the congenitally malformed cochlea. Otolaryngol Head Neck Surg. 1999;121:495-8.

25 Jackler RK, Luxford WM, House WF. Sound detection with the cochlear implant in five ears of four children with congenital malformations of the cochlea. Laryngoscope. 1987; 97:15-7.

26 Shelton C, Luxford WM, Tonokawa LL, Lo WW, House WF. The narrow internal auditory canal in children: a contraindication to cochlear implants. Otolaryngol Head Neck Surg. 1989;100:227-31.

27 Incesulu A, Vural M, Erkam U, Kocaturk S. Cochlear implantation in children with inner ear malformations: report of two cases. Int J Pediatr Otorhinolaryngol. 2002;65:171-9.
28 Casselman JW, Offeciers FE, Govaerts PJ, Kuhweide R, Geldof H, Somers T, et al. Aplasia and hypoplasia of the vestibulocochlear nerve: diagnosis with MR imaging. Radiology. 1997;202:773-81.

29 Kawasaki A, Fukushima K, Kataoka Y, Fukuda S, Nishizaki K. Using assessment of higher brain functions of children with GJB2-associated deafness and cochlear implants as a procedure to evaluate language development. Int J Pediatr Otorhinolaryngol. 2006;70:1343-9.

30 Hamzavi J, Baumgartner WD, Egelierler B, Franz P, Schenk B, Gstoettner W. Follow up of cochlear implanted handicapped children. Int J Pediatr Otorhinolaryngol. 2000;56:16974.

31 Wiley S, Jahnke M, Meinzen-Derr J, Choo D. Perceived qualitative benefits of cochlear implants in children with multi-handicaps. Int J Pediatr Otorhinolaryngol. 2005;69:791-8.

32 Fallon M, Harris M. Training parents to interact with their young children with handicaps: professional-directed and parent-oriented approaches. Infant Toddler Intervent. 1991;1: 297-313.

33 Hadadian A, Merbler J. Parents of infants and toddlers with special needs: sharing views of desired services. Infant-Toddler Interv: J Transdiscipl. 1995;5:141-51.

34 Hoffman RA, Downey LL, Waltzman SB, Cohen NL. Cochlear implantation in children with cochlear malformations. Am J Otol. 1997; 18:184-7.

35 Phelps PD, King A, Michaels L. Cochlear dysplasia and meningitis. Am J Otol. 1994;15: $551-7$. 